\title{
Calibration transfer and drift counteraction in chemical sensor arrays using Direct Standardization
}

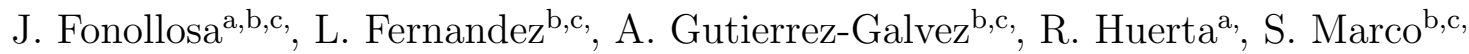 \\ ${ }^{a}$ Biocircuits Institute, University of California San Diego. La Jolla, CA 92093, USA \\ ${ }^{b}$ Institute for Bioengineering of Catalonia. 08028 Barcelona, Spain \\ ${ }^{c}$ Department of Engineering. Universitat de Barcelona. 08028 Barcelona, Spain
}

\begin{abstract}
Inherent variability of chemical sensors makes it necessary to calibrate chemical detection systems individually. This shortcoming has traditionally limited usability of systems based on Metal Oxide gas sensor arrays and prevented mass-production for some applications. Here, aiming at exploring calibration transfer between chemical sensor arrays, we exposed five twin 8-sensor detection units to different concentration levels of Ethanol, Ethylene, CO, or Methane. First, we built calibration models using data acquired with a master unit. Second, to explore the transferability of the calibration models, we used Direct Standardization to map the signals of a slave unit to the space of the master unit in calibration. In particular, we evaluated the transferability of the calibration models to other detection units, and within the same unit measuring days apart. Our results show that signals acquired with one unit can be successfully mapped to the space of a reference unit. Hence, calibration models trained with a master unit can be extended to slave units using a reduced number of transfer samples, diminishing thereby calibration costs. Similarly, signals of a sensing unit can be transformed to match sensor behavior in the past to mitigate drift effects. Therefore, the proposed methodology can reduce calibration costs in mass-production and delay recalibrations due to sensor aging. Acquired dataset is made publicly available.
\end{abstract}

Keywords: Chemical sensors, Calibration transfer, MOX sensors, electronic nose, Direct Standardization, Public Dataset

Email address: jfonollosa@ibecbarcelona.eu (J. Fonollosa) 


\section{Introduction}

Devices composed of an array of unspecific chemical gas sensors coupled with machine learning algorithms have been proposed to solve large diversity of tasks, although such devices found limited use beyond laboratory settings and they are still far from fulfilling industry requirements $[1,2]$. Inherent variability of chemical gas sensors degrades the performance of calibration models when transferred to other sensing systems [3]. Hence, calibration needs to be performed for each system individually, even if the chemical detection platform includes the same number and type of sensors. As a result, mass-production is unfeasible and calibration transfer between systems has been identified as one of the main obstacles towards wide-spread deployment [4].

Unlike sensor drift and robustness, which were studied thoroughly during the last decade $[5,6,7,8,9,10]$, calibration transfer between chemical gas sensor arrays received much less attention from the research community, even though calibration is an expensive and timeconsuming process. An efficient calibration transfer methodology would map the spaces of two sensing systems by means of a small set of transfer samples. Such transformation would enable the use of a calibration model built for one instrument (master) to build another calibration model for the slave instrument. As the number of transfer samples would be smaller than the number of calibration samples, such methodology would reduce the cost of calibrating new systems, thereby alleviating the road to industrial applications for systems based on chemical sensor arrays.

Calibration transfer techniques have been explored in spectroscopic instruments to preserve models in time or update the models after hardware replacement. The different strategies that are found in the literature can be divided in two groups [11]. First, standardization methods attempt at mapping system space at measurement time back to system space during calibration. Direct Standarization (DS) and Piecewise Direct Standardization (PDS) are two prominent examples of methodologies of this group $[12,13]$. Second, other methodologies aim at removing variation in the responses of different instruments (or the same instrument at different measurement times) to cancel out dissimilarity, such as Orthogonal Signal Correction (OSC) and Generalized Least Squares Weighting (GLSW) [14, 15, 16, 17]. The success of calibration transfer techniques made them suitable for being extended to other sensory 
systems.

Pioneering studies on calibration transfer between chemical detection systems were focused on classification problems. In early 2000s, Balaban et al. used two commercially available systems based on polymeric sensors to measure milk samples [18]. They explored three methodologies to transform data acquired with one sensing unit to the space of another unit: Simple coefficient, coefficient with intercept, and a matrix transformation (which actually is equivalent to DS). They found that the latter was the most satisfactory approach. Tomic et al. used five units of a quartz micro balance sensor array [19]. They tested an approach that includes a linear regression to compensate each sensor individually, and a multivariate approach based on partial least squares regression that compensates the whole set of sensors. Both methodologies could successfully remove signal shift, showing slightly better performance the univariate method. In another study, again Tomic et al. attempted calibration transfer for milk sample classification [20]. They used a hybrid device that combined two sensing principles: field-effect transistors and Metal Oxide (MOX) gas sensors. They explored how to compensate the entire replacement of the sensor array that took place between two series of measurements. Multiplicative drift correction and component correction showed similar performance to transfer the signals from the slave unit to the space of the master unit. More recently, Shaham et al. showed the feasibility of mapping responses from sensor arrays composed of different technologies: quartz microbalance and conducting polymeric sensors [21]. They evaluated principal components regression, partial least squares, neural networks and tessellation-based linear interpolation. The best results were obtained with neural networks, although the quality of the result was dependent on the direction of the mapping.

Only recently calibration transfer techniques have been used in regression tasks. In contrast to classification tasks, regression is a more challenging problem, but also offers a more sensitive measure of the quality of the calibration transfer. Lei Zhang et al. presented a methodology for on-line calibration transfer [22]. They built six twin units: a master unit and five slave units. Each unit was composed of four MOX gas sensors along with temperature and humidity sensors. They fit univariate linear regression curves between each of the slave units and the master unit to transform the signals acquired with slave units to the space of the master unit. Although the units were exposed to formaldehyde, benzene and toluene, 
only the former was used as reference for calibration transfer. Their results show that a simple homogeneous linear transformation provides good signal mapping between sensing units. In another study by Deshmukh et al., the authors proposed calibration transfer between two chemical sensor arrays by means of box-behnken design and robust regression [23]. Two twin systems with six MOX gas sensors each were built and tested simultaneously. Artificial neural network models were built with the master unit to predict the concentration of four compounds relevant for the paper industry: hydrogen sulfide, methyl mercaptan, dimethyl disulphide, and dimethyl sulphide. The authors showed that the calibration model developed for the master system, built upon 100 calibration samples, can be transferred to the slave unit using a smaller set of 27 transfer samples, resulting in a faster calibration of the slave system. Finally, in a very recent work, Yan and Zhang developed three twin devices that included eight MOX sensors each [24]. They employed windowed piecewise direct standardization to transform the variables from the slave device to match the master unit. They tested their approach on six regression tasks: acetone, hydrogen or ammonia mixed with synthetic air or exhaled air. Although the complexity of the generated dataset, the authors did not merge different compounds and backgrounds in the tasks, building regression models for one single compound.

The above mentioned approaches confirm the feasibility to map, by means of a set of transfer samples, the signal spaces of different sensing units. This transformation enables the model built with calibration samples acquired with the master unit to be used with the slave device, thereby reducing calibration costs. Nevertheless, previous methodologies that considered regression tasks, to the best of the authors' knowledge, did not explore cross-sensitivity to other volatiles. Basically, although the sensing units were exposed to different volatiles, for each compound, a regressor was trained separately using the samples of the selected compound to quantify its concentration, and the resulting models were not tested with the other volatiles. This can yield to overoptimistic results as the models may predict high concentration levels of the calibrating gas when samples composed of other compounds are presented. Moreover, previous contributions did not consider repetitions of the same sensing unit in time. Hence, whether transferred models degrade in time or whether calibration transfer techniques can be applied to the same device periodically to alleviate drift effects remain open questions. Finally, some of the previous datasets do not 
exactly replicate the scenario of building calibration models for standalone sensing units. Datasets were generated by placing the different units together on the experimental setup, acquiring the data for all the devices (master and slave units) simultaneously. This is not realistic since, in industrial production, one may need to calibrate slave devices while the master unit is not available. As a result, changes in uncontrolled environmental conditions (temperature and relative humidity) may increase sensor response variability between units. In other experimental setups, twin units shared sensor conditioning electronics, and therefore, sensing units cannot be considered completely independent devices.

The goal of the present work is to study calibration transfer between MOX sensor arrays on a realistic scenario that also includes sensor drift. In contrast to previous approaches, our methodology relies on a multiclass regression model that is trained and tested using samples of different compounds, and therefore, incorporates prediction errors due to miss-classification and cross-sensitivity. To target this goal, we generated a complete dataset that was acquired by exposing five stand-alone sensing units to 4 gases at 10 different concentration levels in a period of 22 days. A total of 640 measurements was performed to obtain, to the best of our knowledge, the largest dataset designed for calibration transfer purposes. Furthermore, the acquired dataset is made available to the research community for further study and benchmark of methodologies ${ }^{1}$. The remainder of the paper is organized as follows. We present the experimental setup (Section 2) and the methodology to generate the dataset (Section 3), followed by the results (Section 4) and the conclusions of this work (Section 5).

\footnotetext{
${ }^{1}$ The dataset will be made publicly available upon acceptance of the manuscript
} 


\section{Experimental setup}

\subsection{Detection units}

Conductometric gas sensors, and in particular MOX sensors, are a popular choice due to their cost-efficient design, easy operation, sensitivity, fast response, and number of volatiles that can be detected [25]. Moreover, several options can be found in the market, facilitating sensor integration in arrays. The sensing principle of MOX sensors relies on the absorption or desorption of a gas on the sensitive layer, which induces a change in the sensor conductivity. The composition of the sensing layer and the operating temperature of the sensor, which is usually controlled with a built-in heater, determine its sensitivity to the different volatiles. A MOX sensor operating at a different temperature behaves differently and can be, effectively, considered as a new virtual sensor. Hence, in order to increase the receptive range of detection systems, usually, different types of sensors (and/or sensors working at different temperatures) are included in the arrays. Although different types of sensors are designed to detect different target volatiles, the non-specificity of the sensors makes them sensitive to wide spectrum of analytes.

In order to obtain different sensing units with similar specifications, we implemented five independent, stand-alone chemical detection platforms following the same design. Each platform can hold 8 MOX sensors and integrates custom-designed electronics for sensor control and signal conditioning. In particular, each sensing unit included 4 different types of commercially available sensors (Figaro USA Inc., Glenview, USA) to generate multivariate responses to the different presented stimuli. The control electronics allowed setting the operating temperature of each sensor individually by means of a pulse width modulated signal applied to the heater of each sensor. Table 1 shows the sensor types included in each sensing unit along with the corresponding mean voltage induced in the heater. Note that the repetitions of the same sensor type are operating at different temperatures. As a result, each channel $(\mathrm{CH})$ of the sensing units holds a sensor with unique configuration (sensor type and voltage in the heater). To minimize the variability within the different units, the configuration was repeated in all the units: the sensors of the same type and operating temperature were always placed on the same channel of the respective boards.

In summary, following the same system design and implementation, we designed and built 
5 detection units composed of 8 MOX sensors each.

Table 1: Types of MOX sensors (provided by Figaro Inc.) and corresponding voltage induced in the heater.

\begin{tabular}{|c|c|c|}
\hline Channel & Sensor type & Voltage in sensor heater \\
\hline 0 & TGS2611 & $5.65 \mathrm{~V}$ \\
1 & TGS2612 & $5.65 \mathrm{~V}$ \\
2 & TGS2610 & $5.65 \mathrm{~V}$ \\
3 & TGS2602 & $5.65 \mathrm{~V}$ \\
4 & TGS2611 & $5.00 \mathrm{~V}$ \\
5 & TGS2612 & $5.00 \mathrm{~V}$ \\
6 & TGS2610 & $5.00 \mathrm{~V}$ \\
7 & TGS2602 & $5.00 \mathrm{~V}$ \\
\hline
\end{tabular}

\subsection{Gas mixing station and data acquisition}

Variations in the composition of gas mixtures induce changes in the MOX sensor's conductivity. We developed an experimental setup to acquire continuously the conductivities of a 8-sensor array while the gas conditions are controlled. The complete setup consists of a data acquisition platform, a power control module, and a chemical delivery system. For an accurate and reproducible data generation, the system was fully operated by a computerized environment.

The gas delivery system was based on three independent fluidic branches, each of them controlled by a Mass Flow Controller (MFC) system. The first fluidic branch was used to control the flow of dry air, whereas the other two branches were free to be connected to any pressurized gas cylinder. The gases were supplied by Airgas Inc. in calibrated pressurized gas cylinders. The three branches met together to obtain the desired gas mixtures. MFC were set to induce the desired concentration levels while keeping the total flow at $400 \mathrm{ml} / \mathrm{min}$. The sensor array was placed in a $60-m l$ sealed chamber with 8 openings in its bottom that fit with the standard TO-5 package. Finally, the resulting mixture passed through the measurement chamber continuously before being collected by the exhaust system. The sensors' conductivities were acquired continuously at $100 \mathrm{~Hz}$ throughout the complete experiment. 


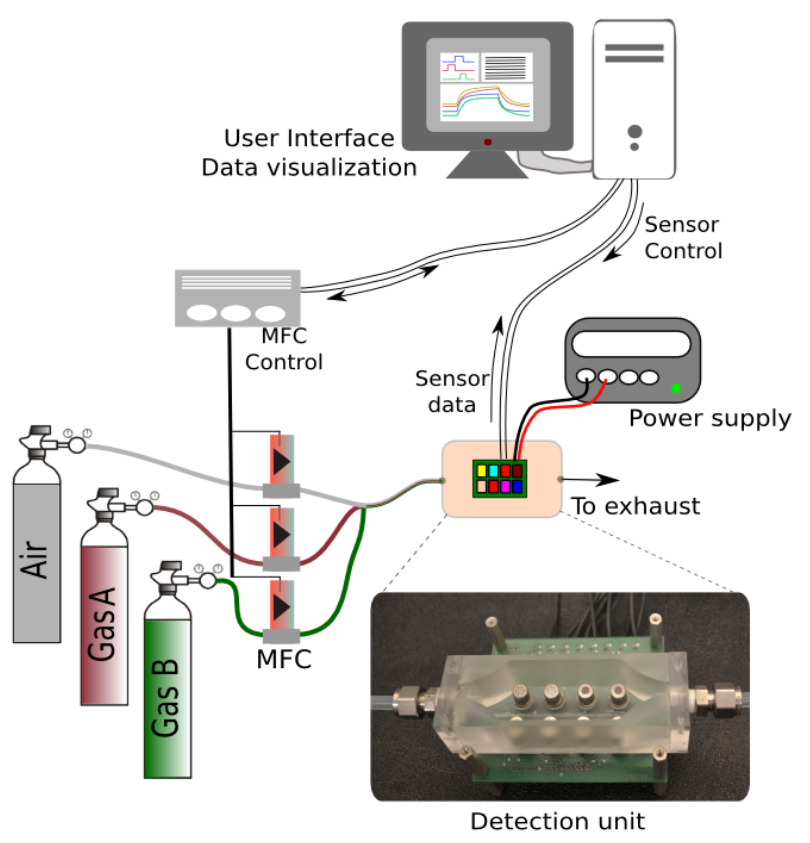

Figure 1: Experimental setup with one of the detection units. By means of a set of three MFC the total flow was kept constant at $400 \mathrm{ml} / \mathrm{min}$ in the measurement chamber, while different concentration levels of Ethanol, Methane, Ethylene, and Carbon Monoxide were presented to the sensor unit. We built 5 standalone sensor units following the same design. Each unit is composed of eight MOX sensors and was tested individually.

Figure 1 shows a diagram of the experimental setup along with one of the implemented detection units.

Hence, by means of a set of three MFCs and the acquisition system, the sensor array was exposed to controlled gas conditions, while the sensors' conductivities were recorded. At the end of the measurement, we acquired 8 time-series that were indicative of the presented gas conditions.

\subsection{Experimental protocol}

The same experimental protocol was followed to measure the response of the 5 chemical detection platforms. Each day, a different unit was tested, which included the presentation of 40 different gas conditions, presented in random order. In particular, the board under test was exposed to 10 concentration levels of Ethanol, Methane, Ethylene, and Carbon Monoxide. The gas mixtures were generated with calibrated gas cylinders at different concentrations: 1000 ppm for Carbon Monoxide and Methane, and 500 ppm for Ethylene and Ethanol. Table 2 shows the tested concentration levels for each volatile. Moreover, the sensory units were 
Table 2: Tested analytes and corresponding concentration setpoints. Each volatile was presented at 10 concentration levels.

\begin{tabular}{|c|c|}
\hline Analyte & Concentration levels $(\mathrm{ppm})$ \\
\hline Ethylene & $12.5,25.0,37.5,50.0,62.5,75.0,87.5,100.0,112.5,125.0$ \\
Ethanol & $12.5,25.0,37.5,50.0,62.5,75.0,87.5,100.0,112.5,125.0$ \\
Carbon Monoxide & $25.0,50.0,75.0,100.0,125.0,150.0,175.0,200.0,225.0,250.0$ \\
Methane & $25.0,50.0,75.0,100.0,125.0,150.0,175.0,200.0,225.0,250.0$ \\
\hline
\end{tabular}

Table 3: Days in which each detection platform was tested.

tested several times over a period of 22 days (see Table 3 ).

\begin{tabular}{|c|c|}
\hline & Days tested \\
\hline Unit 1 & $4,10,15,21$ \\
Unit 2 & $1,7,11,16$ \\
Unit 3 & $2,8,14,17$ \\
Unit 4 & 3,9 \\
Unit 5 & 18,22 \\
\hline
\end{tabular}

The design of the experiment was the same for the four tested volatiles: First, a constant flow of air (carrier gas) circulated through the sensing chamber for $50 \mathrm{~s}$. This step constitutes a preliminary stabilization phase, which served to measure the baseline of the sensor response. Second, the carrier gas was mixed with the selected volatile at the desired concentration level. The resulting gas mixture circulated during $100 \mathrm{~s}$. Finally, the vapor was purged out from the test chamber by re-circulating only clean air during the subsequent $450 \mathrm{~s}$. Therefore, the total duration of each experiment was $600 \mathrm{~s}$. Once the recovery phase was complete, a new measurement could restart. The order of the concentration levels and the order of the tested volatiles were selected randomly for each experiment and day.

In summary, the acquired dataset, which was generated over the course of 22 days, includes 640 different measurements, distributed in five sensing units exposed to four volatiles at ten different concentration levels each volatile. 


\section{Methodology}

\subsection{Calibration models}

We evaluated the ability of calibration models built with one chemical detection unit (master) to predict the concentration level of samples presented to other (slave) sensing units. In order to build the calibration models, we concatenated the response of the 8 sensors contained in the same unit. The acquired signals were downsampled to 300 samples per measurement to alleviate computational costs. This would be equivalent to acquire the sensors' responses at a sampling frequency of $0.5 \mathrm{~Hz}$. Hence, for each measurement, we concatenated 2400 features in a vector.

The calibration models were trained to identify the presented compound and its concentration level. Using functions in scikit-learn library [26], we built two-layer calibration models: First, a Support Vector Machine (SVM) classifier was trained to predict the gas type presented to the sensor array. The models were trained to classify the four different classes (gas types) using one-versus-all approach. We opted for SVM-based classifiers due to their proven success in gas classification problems [27]. Moreover, in a previous study that we carried out using the same design for the detection unit, we explored the ability of different classifiers to predict the presence-absence of ethylene in different backgrounds [28]. LDA, k-NN, Perceptron, and SVM did show similar classification accuracy when trained and tested under the same conditions. However, SVM classifiers seemed to show higher flexibility to correctly classify samples at lower concentration levels than the calibration samples. Other works that explored several models to classify data from MOX sensor arrays also result in similar performances for different models. For example, detection of potato soft rot was explored using a set of 12 MOX gas sensors. Predictive models based on LDA, MARS, RBF SVM, Random forests and C5.0 were built. With similar performance, RBF SVM and LDA appeared to be the best suited models for early discrimination of healthy controls from infected samples [29].

Second, we trained four Support Vector Regression (SVR) to estimate the concentration of each compound. The output of the classifier determined which regression model was employed to estimate the concentration of an unknown sample. Therefore, the predicted output, as well as the presented sample, can be represented in a 4-component vector, which 
is a convenient representation to compute prediction error. In fact, the error of the predicted sample was computed by means of the Euclidean distance from the presented sample:

$$
\text { Error }=\operatorname{dist}\left(\vec{x}_{\text {presented }}, \vec{x}_{\text {predicted }}\right)
$$

We selected one sensing unit as master device and we utilized the measurements performed in one day to build a calibration model. From the 40 available measurements, we selected 20 measurements as calibration samples. In particular, the concentration levels 2,4,6,8, and 10 for each of the compounds were used to build the calibration model. Therefore, the classifier was trained with 20 calibration samples, and 5 samples were utilized to train each regression model. The models were trained such that a 5-fold cross-validation error was minimized. The rest of the concentration levels $(1,3,5,7$, and 9$)$ were set aside to test the performance of the model within the same board and repetition (test samples). In order to perform the calibration transfer between sensing units (transfer samples), we only considered a subset of the calibration samples: concentration levels 4 and 8. Therefore, in contrast to the master unit which is calibrated using 20 calibration samples, a slave unit would be calibrated using only 8 transfer samples (2 from each compound). This represents a $60 \%$ reduction of the required number of samples to calibrate a new unit.

\subsection{Direct Standardization}

Direct Standardization is a multivariate transfer technique by which one sample in the new space (sample measured with the uncalibrated system) is mapped to the reference space (same sample measured with the reference instrument). The relationship of the transformation is given by:

$$
S_{\text {master }}=S_{\text {slave }} F
$$

where $S_{\text {master }}$ and $S_{\text {slave }}$ are the response matrices of the transfer samples (also called standardization samples) acquired with the master and the slave devices. Both response matrices have as many rows as transfer samples used to perform the transformation, and as many columns as the number of features of each sample. Then, the transformation matrix F can be estimated from the pseudo-inverse matrix of the transfer samples. Finally, new samples can be transformed to the reference space by means of the transformation matrix. 
DS assumes that all the variations in the signals are caused by the variability of the sensing devices. However, since the experiment setups to deliver chemical samples also suffer from some uncertainty, any variation in the sample concentration will be incorporated in the transformation matrix as well. Moreover, DS also assumes a linear relationship between the signals from both instruments. Despite such limitations, DS is a transfer technique that has been successfully applied to near-infrared spectroscopy. The reader is referred to the literature for more details $[30,31]$. 


\section{Results}

\subsection{Sensor variability}

For each measurement, we acquired 8 signals that embody the response of the sensor array to the presented gas conditions. Figure 2 shows that the acquired signals indeed follow the changes in the composition of the gas sample. Moreover, different stimuli induce different sensors' responses.

Although the design of the detection platforms is the same for all the boards, the variability of sensors disperses the responses of the sensing units. Note, for example, that baseline shifts are more prominent between different boards (see Fig. 2b) than the baseline shifts within the same board (see Fig. 2a), even if the experiments were more distant in time (17 days compared to two consecutive days). Also, the sensitivity of the sensors of the same type varies from one unit to another (see channels 4,5,6 in Fig. 2b). Therefore, from a simple visual inspection of the acquired signals, one can confirm the variability of the sensors, both across units of the same type and across time.

We utilized the model of Clifford-Tuma [32,33] to quantify the sensor variability. Based on experimental observations, the model describes the sensor resistance $\left(R_{S}\right)$ as a function of the gas concentration $(c)$ and the sensor resistance in air $\left(R_{0}\right)$. At operating temperature, the model can be simplified to $[34,35]$ :

$$
\log \left(\frac{R_{0}-R_{S}}{R_{S}}\right)=\log (s)+\beta \log (c)
$$

where both, $s$ and $\beta$ are parameters that depend on the analyte under test and the sensor's operating temperature.

Using the ten concentration levels of each compound that we acquired each day for each sensor, we fitted the sensors' responses according to Eq. 3. Therefore, for each sensor, we obtained 64 pairs of values that are indicative of the variation on the sensor's behavior across units and across time. Figure 3 shows the estimated value for $\beta$ for the five sensors placed in the channel 3 of the sensing boards. The fitted functions for the five sensors are also presented for $\mathrm{CO}$ and Ethylene. The sensitivity to the different volatiles can be considered as the chemical signature of the sensor. However, from Fig. 3 one can conclude that the 

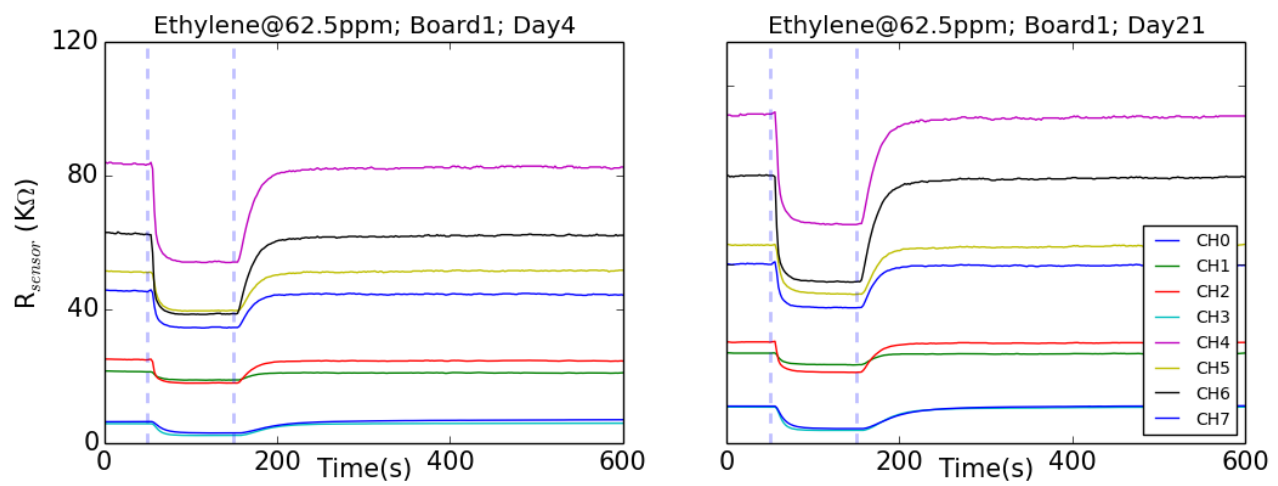

(a) Reproducibility within the same unit
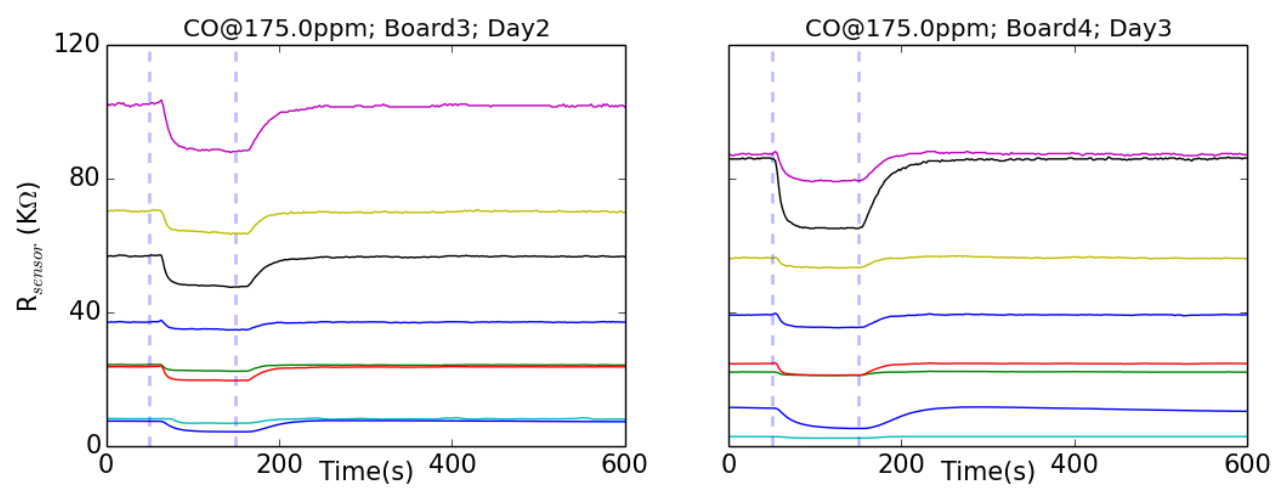

(b) Reproducibility within different units

Figure 2: Acquired time series for different detection units under different gas conditions. The sensors are able to follow the changing gas conditions (dashed vertical lines indicate start/stop of selected gas release). Each volatile induces a different response to the sensor array. Although the design of each sensing unit is the same, the acquired signals differ significantly from one board to another (note prominent baseline shifts and different sensor sensitivities to the same stimuli in the bottom panel). 

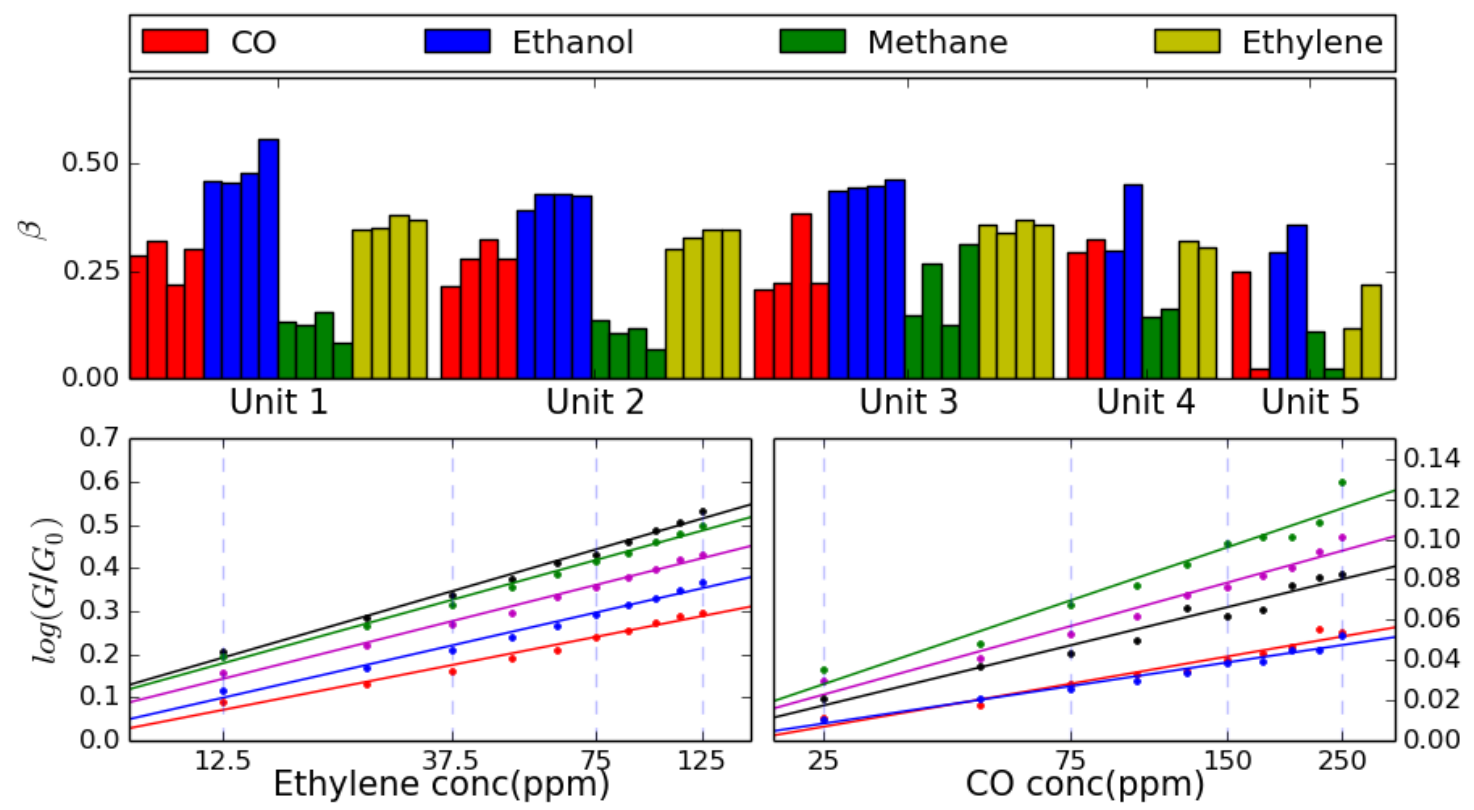

Figure 3: Clifford-Tuma model was used to fit acquired data and estimate $s$ and $\beta$ for each sensor and volatile. Top panel shows $\beta$ for the five sensors of the same type placed in the channel $\mathrm{CH} 3$ of the five units. Each sensor is identified by the unit it belongs to (see label Unit $1-5$ ). Parameter $\beta$ is presented for the four gases (grouped in colors) and for the number of repetitions of each condition. Bottom panels show fitted models along with acquired data, confirming the quality of the fit. Each color represents a different sensor of the same type. The slope of each linear fit corresponds to a $\beta$ value presented in the top panel.

behavior of the sensors changes across boards and also within the same sensor, since each repetition was measured days apart.

\subsection{Signal mapping}

We collected data with a master unit and compared the acquired data with the signals from a slave unit exposed to the same conditions. Figure 4 shows the captured signals with the master unit and the slave unit when Ethylene at 10 concentration levels was presented. From a visual inspection, one can confirm the variability between captured signals for sensors of the same type: For example, the steady state corresponding to the highest concentration level $(125 \mathrm{ppm})$ for the master unit would correspond to a much lower concentration level (50 ppm) for the slave unit. This mismatch between sensors' sensitivities would limit the prediction ability of the calibration models. However, after applying DS, with only two transfer samples, the responses captured with the slave unit are successfully mapped to the space of the master unit. 


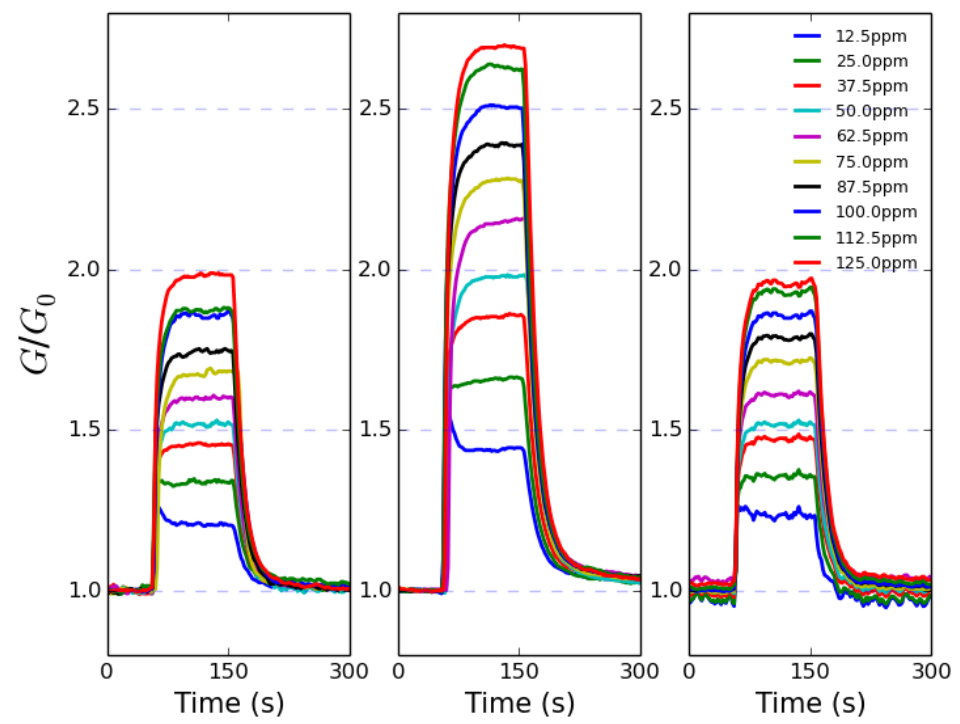

Figure 4: Signals captured for a sensor of the same type (CH7) using a master unit (left) and a slave unit (middle) exposed to Ethylene at different concentration levels. The sensitivity of the sensors differs significantly from unit to unit. The signals captured with the slave unit after DS transformation (right) are mapped to the signals captured with the master.

To visualize the captured signals before and after DS transformation, and compare the signal spaces of slave and master unit, we projected captured signals using Principal Component Analysis (PCA) for data visualization at a lower dimension space. We applied PCA on the data captured with a master unit (see Fig. 5). The first two Principal Components for the master unit were plotted. Then, using the same data projection, data acquired with a slave unit was added to the plot. One can conclude that the data is grouped in clusters for each compound. Every cluster spreads out from a region that represents clean air to higher concentration levels. Moreover, data acquired with the slave unit results in clusters with different sizes and angles from the origin. However, after DS transformation, the data from slave unit is better aligned with respect to the master datapoints. This confirms the ability of DS to map slave data to the space of a master unit using only two transfer samples per volatile.

\subsection{Calibration transfer}

\section{Transfer between devices}

We built a calibration model selecting a sensing unit as master device and using only cal- 


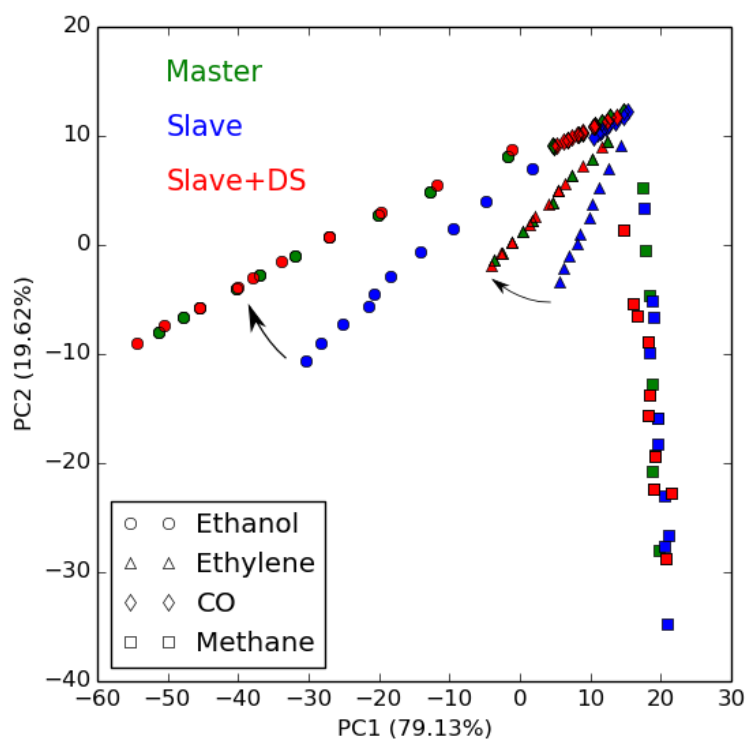

Figure 5: PCA transformation of the signals captured with the master unit (green). Signals from the slave unit (blue) are represented using the same projection. After DS, data captured with the slave unit (red) is successfully transformed to the space of the master unit.

ibration samples. The prediction ability of the model was evaluated using the test samples that were acquired the same day with the master. We also evaluated the calibration models with the test samples but acquired with the rest of the devices (slave). We repeated the process until all the units and repetitions were selected to build a calibration model (master) and tested with the rest of the devices and repetitions. For each configuration, we computed the cross validation error (internal error in training), the error with the test samples of the same unit and day, and the error with the samples acquired with other units. To evaluate the error with other units, we input the raw signals as they were acquired and the signals after DS transformation. Figure 6 shows the distributions of obtained prediction errors after 200 repetitions (samples acquired with unit 5 when exposed to methane were discarded as acquisition issues resulted in corrupted signals). Since the ranges of presented concentrations were not the same for each volatile, errors in the volatiles that were presented at higher concentrations bias the total prediction error. In order to balance the contribution of each volatile to the prediction error, the prediction errors were normalized to the maximum concentration level presented for each gas. Therefore, in Fig. 6, prediction errors are presented as a relative error with respect to the maximum concentration for each volatile. Table 4 details the first 
and third quartiles of the distribution of errors in ppm for each volatile.

Several conclusions can be drawn from Fig. 6 and Table 4. First, the trained models show good generalization since the differences between the error in internal validation and the error with test samples are not significant. Second, the ability of the calibration models to predict the concentration of new samples decays when the measurements are performed with other sensing units. Although for some repetitions one still obtains errors in the predictions of the same order than when using the master unit, there are noticeable occasions in which the errors increase dramatically. These high concentration errors result in uncertainty on the quality of the prediction in test. However, after a DS transformation, using only two transfer samples, the prediction ability is improved. Actually, prediction errors after DS transformation are comparable to the errors obtained with test samples (with the master unit). The median of the error decreases from $17 \%$ to $4.7 \%$ when DS is applied. The latter is only slightly higher than the error obtained measuring the same day with the master: $3.9 \%$, which provides a performance limit for the prediction accuracy of the models.

In order to confirm the flexibility of our approach, we evaluated the same methodology using a different calibration model and several calibration transfer techniques. First, we used Principal Least Squares (PLS) instead of SVR models. Obtained classification accuracies showed that SVR outperforms PLS when testing the models with the same unit (12\% for PLS versus 3.9\% for SVR). However, the accuracy of the predictions when the models are transferred to another unit becomes similar for both classifiers (19.2\% for PLS, and $17.0 \%$ for SVR). This result indicates that the variability between boards is prominent with respect to the choice of the regression model. Finally, when DS was introduced to perform data transfer, accuracies similar to same-unit same-day predictions were recovered: $12.6 \%$ for PLS and $4.7 \%$ for SVR. This confirms the ability of DS to recover the prediction accuracy of different classifiers when extended to other boards.

Second, we explored the ability of other calibration transfer techniques. In particular, we evaluated PDS, OSC, and GLSW that are already implemented in the PLS_Toolbox [36]. Following the same methodology, and using SVR regression, we compared the obtained accuracy to DS. Table 5 shows first/third quartiles of the distribution of errors in ppm for each volatile. Prediction errors for the tested calibration transfer strategies show that the selection of the strategy does not change dramatically prediction accuracies. Best accuracy 


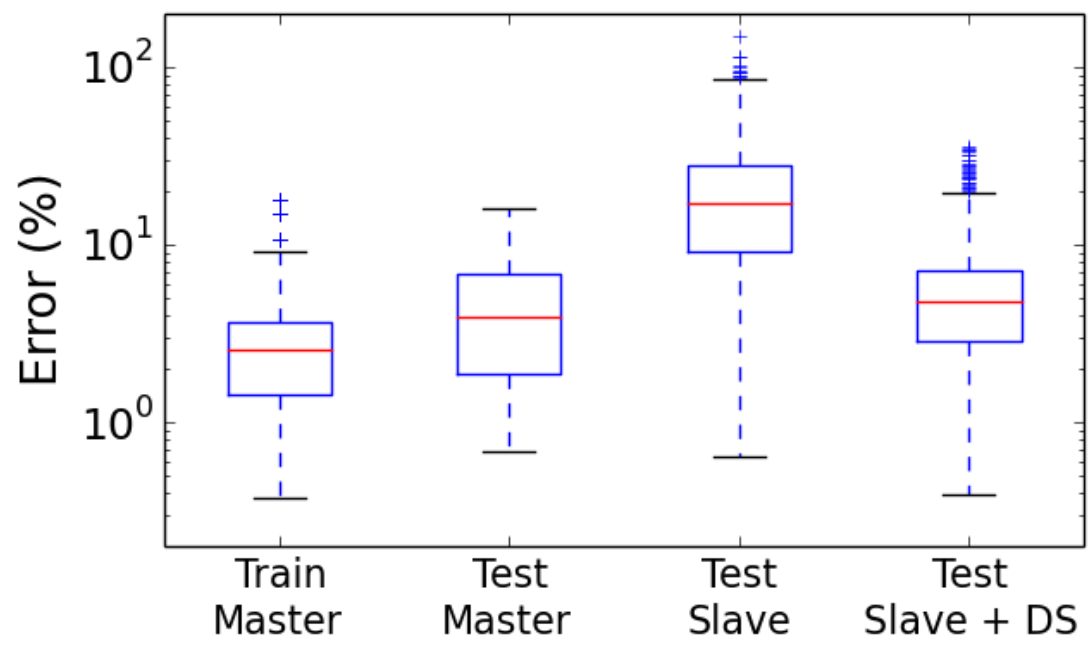

Figure 6: Prediction error distribution of the calibration models when evaluated with data from master/slave units. Error in training (internal validation using samples from the master unit). Prediction error using unseen test samples acquired with the master unit. Prediction error testing samples acquired with slave units, before and after DS transformation. Errors were normalized to the highest concentration level presented for each volatile. Whiskers represent the limit for samples that are further than 3 times the interquartile range from the lower/upper quartiles.

predictions were obtained with DS and PDS. Although the simplicity of DS transformation and its rapid execution in a microprocessor, results confirm the ability of DS to map two sensor spaces.

\section{Transfer within the same device}

We tested whether DS can be used to alleviate sensor drift. Similarly to results presented in Fig. 6, we applied the signal transformation to measurements acquired with the same unit but measured days apart. In particular, we built the calibration models with the calibration

Table 4: Prediction errors for each volatile in training, testing on the same unit, testing on slave units, and testing on slave units after DS. [First Quartile, Third Quartile]

\begin{tabular}{|l|c|c|c|c|}
\hline \multicolumn{1}{|c|}{$(\mathrm{ppm})$} & CO & Ethanol & Ethylene & Methane \\
\hline Train & {$[7.8,19]$} & {$[2.6,4.3]$} & {$[1.6,3.4]$} & {$[2.9,5.8]$} \\
\hline Same unit & {$[11,21]$} & {$[2.3,9.3]$} & {$[2.2,3.4]$} & {$[3.2,8.1]$} \\
\hline Other unit & {$[36,97]$} & {$[16,40]$} & {$[12.5,32]$} & {$[9.9,29]$} \\
\hline Other unit + DS & {$[13,27]$} & {$[4.7,9.4]$} & {$[2.7,5.5]$} & {$[5.2,14]$} \\
\hline
\end{tabular}


Table 5: Prediction errors for each volatile, testing on slave unit after different transfer strategies. [First Quartile, Third Quartile]

\begin{tabular}{|l|c|c|c|c|}
\hline$(\mathrm{ppm})$ & CO & Ethanol & Ethylene & Methane \\
\hline DS & {$[13,27]$} & {$[4.7,9.4]$} & {$[2.7,5.5]$} & {$[5.2,14]$} \\
\hline PDS & {$[12,29]$} & {$[4.6,11]$} & {$[2.7,5.3]$} & {$[5.2,13]$} \\
\hline OSC & {$[14,40]$} & {$[6.5,15]$} & {$[4.2,12]$} & {$[6,14]$} \\
\hline GLSW & {$[15,36]$} & {$[3.5,11]$} & {$[3.4,7.4]$} & {$[9,16]$} \\
\hline
\end{tabular}

samples acquired one day (one unit). To quantify the accuracy of the prediction models, we tested the models with test samples acquired the same day than the calibration samples, and also with samples acquired other days. Figure 7 shows prediction errors in training and using samples acquired other days. One can conclude that DS transformation results in prediction error drop.

We also evaluated the feasibility of a transferred calibration model to be transferred again. In other words, we explored whether calibration models can be concatenated in a sequential chain of calibration transfers. To test multiple transfer of calibrations, we selected a master unit (Unit \#3) and a slave unit (Unit \#1). First, the calibration model built with the master was transferred to the slave unit. Second, the calibration model was transferred again to compensate drift within the same slave unit. Figure 8 shows the prediction error of the slave unit when the master calibration model is used to predict new samples, with and without DS transformation. The prediction error is significantly increased when the model is directly used to evaluate samples from another board, and it remains at approximately $30 \%$ for all the tested time period. However, if the signals are mapped by means of DS transformation, the prediction errors remain of the same order than in the master unit. Therefore, master calibration models can be transferred to slave units, which in turn can be transferred at desired time intervals to counterattack sensor drift.

\section{Continuous monitoring applications}

In the previously explored scenarios, either when calibration transfer was evaluated between different sensing units or within the same unit, we considered that the complete signals were available. However, such assumption is not realistic for continuous monitoring applications, in which the sensing unit is measuring continuously the environment and a reference 


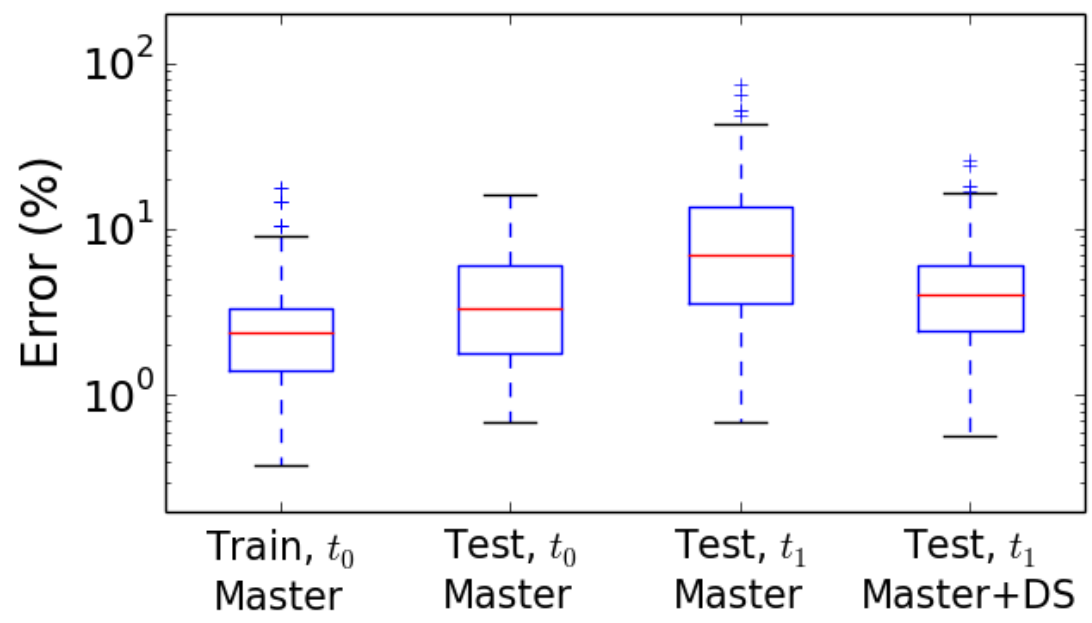

Figure 7: Prediction error distribution of the calibration models when evaluated with samples from the same unit acquired at different times. The sensor array, after some time, can be considered as another virtual device with deviated specifications with respect to the original system. Error in training (internal validation using training samples). Prediction error using unseen test samples acquired the same day than the training samples. Prediction error testing samples acquired days apart (before and after DS transformation). Errors were normalized to the highest concentration level presented for each volatile. Whiskers represent the limit for samples that are further than 3 times the interquartile range from the lower/upper quartiles.

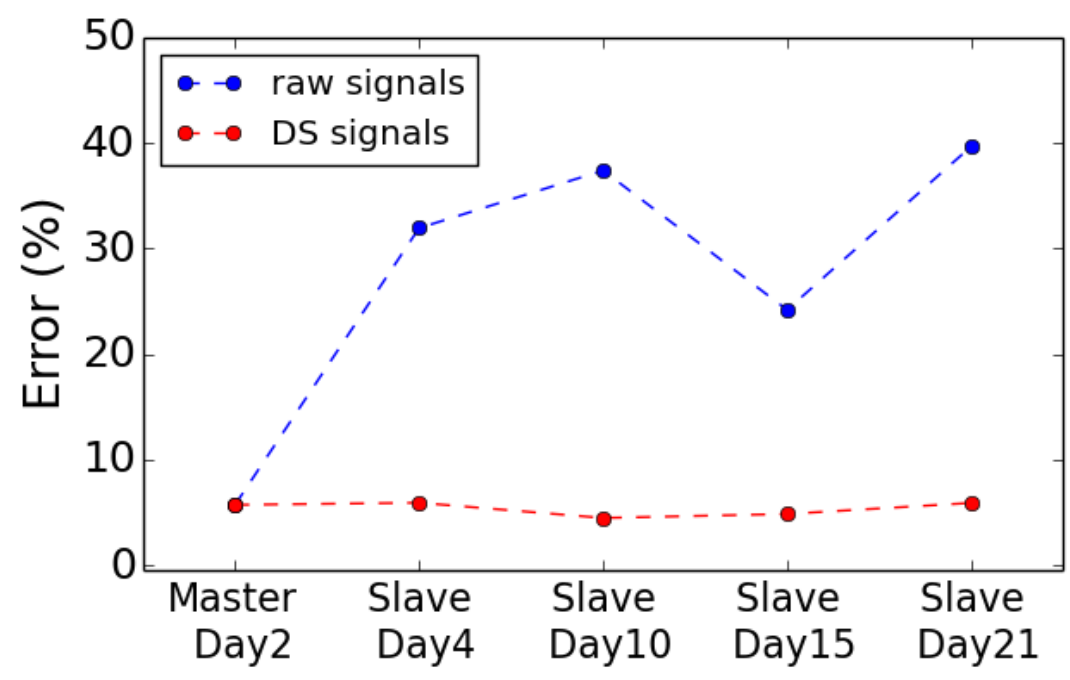

Figure 8: Prediction error when the master calibration model is transferred several times. The master model is transferred to a slave unit, and the resulting model is transferred again to mitigate drift effects. When the signals are mapped with a DS transformation, the prediction errors retain the error of the master unit. 


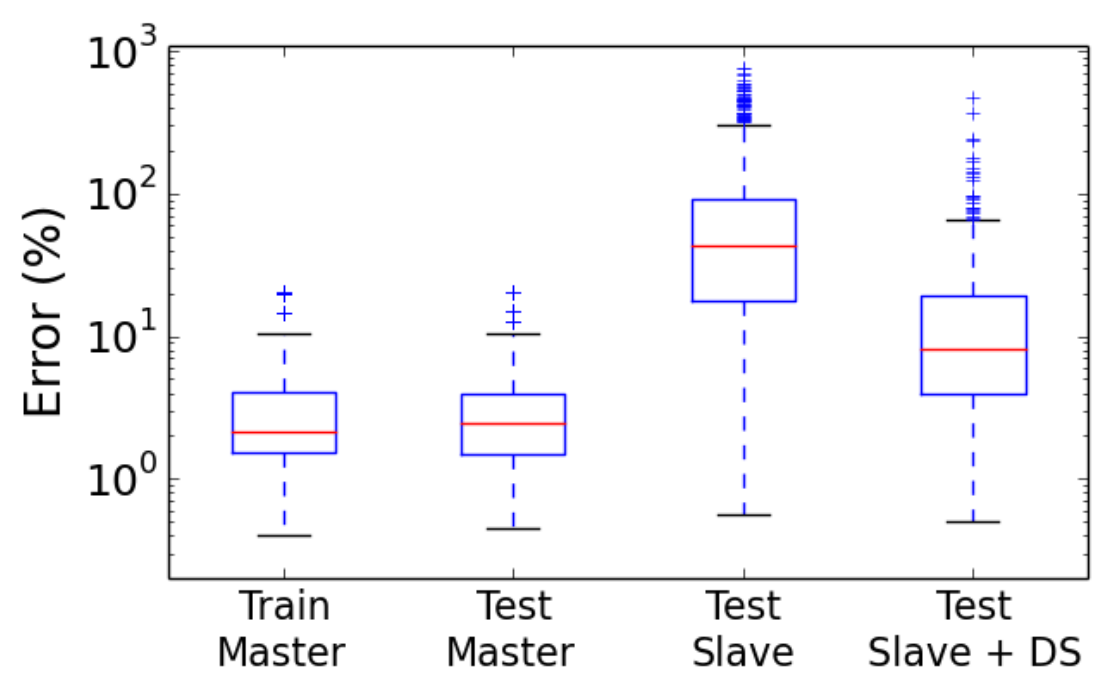

Figure 9: Prediction error distribution of the calibration models for continuous monitoring scenarios. Baseline and transient parts of the signal are not considered. Error in training (internal validation using samples from the master unit). Prediction error using unseen test samples acquired with the master unit. Prediction error testing samples acquired with slave units, before and after DS transformation. Errors were normalized to the highest concentration level presented for each volatile. Whiskers represent the limit for samples that are further than 3 times the interquartile range from the lower/upper quartiles.

is not presented before measuring a new sample. Therefore, baseline and transient portions of the signal are not acquired systematically at the beginning of each new measurement. To simulate monitoring tasks, we only considered the steady state portion of the signal and we repeated the same methodology to evaluate the calibration transfer between units. Figure 9 shows prediction errors when baseline and transient portions of the signal are omitted. It can be seen that the prediction errors increase up to 8 times the highest concentration level. Although DS transformation reduces prediction error, the calibration models do not guarantee an accuracy within the same order of magnitude of the presented concentrations. 


\section{Discussion and conclusions}

We built five twin sensing units that included eight MOX gas sensors each. We exposed the units to the same chemical conditions such that each day the device under test was exposed to 40 mixtures of air with Ethylene, CO, Ethanol, or Methane at 10 concentration levels each. The result is a dataset that is unique since it was generated with 5 standalone sensing units that were exposed to the gas conditions separately, with several repetitions across time. Therefore, the generated dataset is suitable to study sensor diversity, calibration transfer techniques between units and time drift effects. Moreover, the dataset is made publicly available.

We explored sensor tolerance by fitting sensor responses to the Clifford-Tuma model and comparing the obtained sensitivities. Our results show a mismatch between the responses of sensors of the same type. In particular, sensor mismatch becomes prominent in baseline shifts. Actually, such sensor variability is confirmed by the sensor manufacturer. MOX gas sensors provided by Figaro [37] exhibit significant tolerance on sensor resistance measured in air (baseline) and sensitivity: According to the TGS2602 data sheet, for example, sensor baseline ranges between $10 K \Omega-100 K \Omega$ and the relative change in sensor resistance at $10 \mathrm{ppm}$ of Ethanol ranges between $0.15-0.5 \Omega / \Omega$.

Our results showed that the mismatch between sensors limits calibration models transferability from a master unit to a slave unit. We built hierarchical calibration models that included a classifier to determine the gas type and regressors to estimate the concentration level of the presented chemical compounds. Such calibration models allowed us to quantify the quality of calibration transfer considering the combined task of compound identification and concentration quantification. We found that calibration models become inefficient when are used to evaluate samples acquired with other units. However, DS transformation successfully maps slave signals to the space of the master unit, thereby enabling calibration transfer between units.

We showed that DS successfully applies a signal transformation across twin units and within the same unit measuring days apart. This result confirms that calibration transfer techniques can be also applied to compensate drift. Actually, a device after some time can be considered as another virtual device with deviated specifications with respect to the original 
system. Hence, one can easily adopt calibration transfer strategies to counteract drift. We also showed that calibration transfers can be concatenated and that slave units preserve, with no significant increase, prediction errors of the master. This scenario of multiple calibration transfers would be very desirable for mass production of units: One could train calibration models, which could be transferred between units or within the same unit at different times. As a result, a network of standards keeping track of the distance in terms of the number of calibration transfers performed from the original master would be available.

Although DS applies lineal transformation to map signals between units, non-linear behavior captured by the calibration model trained with the master unit is still being transferred to the calibration model for the slave unit. We remark, that in fact, calibration models that we trained are non-linear (SVM with RBF kernels, for example). We explored the accuracy of the transferred models with two transfer samples per compound. Although the number of transfer samples could be increased, the cost of the calibration for the slave unit would be higher and the expected improvement in the performance is marginal. Actually, in our dataset, as shown in the PCA decomposition, the samples corresponding to each gas appear aligned. Therefore, two points from the master and the slave spaces will suffice to map the signals to the new space. Hence, although the simplicity of DS, we showed that it can be used to transfer calibrations between devices and it can help to mitigate drift effects, thereby delaying recalibration of the devices as well. However, sensor systems with higher non-linear behavior or larger variability between units may need larger number of transfer samples to map sensor spaces using a more dense grid of transfer samples.

We also explored continuous monitoring scenario by considering only steady state portion of the signal. The results showed that one needs to include a reference measurement (clean air, for example) before sample presentation to successfully map the signals from the slave unit to the master space. To deal with continuous inputs, regressors with tapped-delayed or non-linear transformation of temporally varying input signals to a higher dimension have been proposed [38, 39, 40,41, 42]. A route to apply calibration transfer in devices that sample continuously would be the combination of such approaches with DS transformation.

Finally, we tested the performance of different popular methods for calibration transfer, namely DS, PDS, OSC, and GLSW. Our results indicate that, although the selection of the methodology does not change classification accuracy dramatically, DS and PDS are the 
methods that provide best calibration transfer among units. This result is in agreement with recent studies on calibration transfer based on MOX sensor arrays [43] and NIR spectroscopy database [11]. In the mentioned works, DS or PDS also outperform other explored calibration transfer methodologies. This may yield to think that DS, or PDS, are the best calibration transfer techniques. However, other calibration transfer techniques may provide better results when examined on sensing systems with different cross-sensitivity among their sensing elements, in calibrations performed with higher number of training samples or training conditions, or when the number of transfer samples becomes larger. The best choice for calibration transfer is probably database sensitive and providing guidelines for its selection requires further study on databases, acquired with sensory systems of different technologies and for different classification or regression tasks.

All in all, for a successful calibration transfer, one needs to acquire transfer samples with the slave unit. Provided that the calibration model for the master unit is already available, slave units trained with smaller set of transfer samples coupled with DS provide similar prediction error than when they are trained with all the set of calibration samples. The lower number of samples needed to build a calibration model will reduce the cost of calibration of new units, alleviating thereby industrial costs. Moreover, calibration transfer techniques could be coupled with other calibration methodologies that aim at reducing calibration costs by selecting the best training examples, or with active sampling strategies to adapt operating sensor temperature to mirror sensor behaviors $[44,45,46]$.

\section{Acknowledgments}

This work was partially funded by the Spanish MINECO program, under grants TEC201459229-R (SIGVOL) and PCIN-2013-195 (SENSIBLE), and from the European Community's ENIAC Joint Undertaking program, under Grant agreement no. 621272 (SAFESENS). JF, LF, AGG, and SM are part of a consolidated research group recognized by the Generalitat de Catalunya (2014-SGR-1445). JF acknowledges grant 2013 Beatriu de Pinós-B 00190. We thank Dr. Emre Neftci for his helpful comments and reviewing the manuscript. 
[1] S. Marco, The need for external validation in machine olfaction: emphasis on healthrelated applications, Analytical and bioanalytical chemistry (2014) 1-16.

[2] P. Boeker, On electronic nose methodology, Sensors and Actuators B: Chemical 204 (2014) 2-17.

[3] S. Deshmukh, R. Bandyopadhyay, N. Bhattacharyya, R. Pandey, A. Jana, Application of electronic nose for industrial odors and gaseous emissions measurement and monitoringan overview, Talanta 144 (2015) 329-340.

[4] S. Marco, A. Gutiérrez-Gálvez, Signal and data processing for machine olfaction and chemical sensing: a review, Sensors Journal, IEEE 12 (11) (2012) 3189-3214.

[5] J.-E. Haugen, O. Tomic, K. Kvaal, A calibration method for handling the temporal drift of solid state gas-sensors, Analytica chimica acta 407 (1) (2000) 23-39.

[6] A. Ziyatdinov, S. Marco, A. Chaudry, K. Persaud, P. Caminal, A. Perera, Drift compensation of gas sensor array data by common principal component analysis, Sensors and Actuators B: Chemical 146 (2) (2010) 460-465.

[7] M. Padilla, A. Perera, I. Montoliu, A. Chaudry, K. Persaud, S. Marco, Drift compensation of gas sensor array data by orthogonal signal correction, Chemometrics and Intelligent Laboratory Systems 100 (1) (2010) 28-35.

[8] A. Vergara, S. Vembu, T. Ayhan, M. A. Ryan, M. L. Homer, R. Huerta, Chemical gas sensor drift compensation using classifier ensembles, Sensors and Actuators B: Chemical 166 (2012) 320-329.

[9] J. Fonollosa, A. Vergara, R. Huerta, Algorithmic mitigation of sensor failure: Is sensor replacement really necessary?, Sensors and Actuators B: Chemical 183 (2013) 211-221.

[10] E. Martinelli, G. Magna, A. Vergara, C. Di Natale, Cooperative classifiers for reconfigurable sensor arrays, Sensors and Actuators B: Chemical 199 (2014) 83-92.

[11] B. M. Wise, R. T. Roginski, A calibration model maintenance roadmap, IFACPapersOnLine 48 (8) (2015) 260-265. 
[12] Y. Wang, D. J. Veltkamp, B. R. Kowalski, Multivariate instrument standardization, Analytical chemistry 63 (23) (1991) 2750-2756.

[13] Z. Wang, T. Dean, B. R. Kowalski, Additive background correction in multivariate instrument standardization, Analytical Chemistry 67 (14) (1995) 2379-2385.

[14] H. Martens, T. Naes, Multivariate calibration, John Wiley \& Sons, 1992.

[15] S. Wold, H. Antti, F. Lindgren, J. Öhman, Orthogonal signal correction of near-infrared spectra, Chemometrics and Intelligent Laboratory Systems 44 (1) (1998) 175-185.

[16] J. Sjöblom, O. Svensson, M. Josefson, H. Kullberg, S. Wold, An evaluation of orthogonal signal correction applied to calibration transfer of near infrared spectra, Chemometrics and Intelligent Laboratory Systems 44 (1) (1998) 229-244.

[17] H. Martens, M. Høy, B. M. Wise, R. Bro, P. B. Brockhoff, Pre-whitening of data by covariance-weighted pre-processing, Journal of Chemometrics 17 (3) (2003) 153-165.

[18] M. Balaban, F. Korel, A. Odabasi, G. Folkes, Transportability of data between electronic noses: mathematical methods, Sensors and Actuators B: Chemical 71 (3) (2000) 203211.

[19] O. Tomic, H. Ulmer, J.-E. Haugen, Standardization methods for handling instrument related signal shift in gas-sensor array measurement data, Analytica Chimica Acta 472 (1) (2002) 99-111.

[20] O. Tomic, T. Eklöv, K. Kvaal, J.-E. Haugen, Recalibration of a gas-sensor array system related to sensor replacement, Analytica Chimica Acta 512 (2) (2004) 199-206.

[21] O. Shaham, L. Carmel, D. Harel, On mappings between electronic noses, Sensors and Actuators B: Chemical 106 (1) (2005) 76-82.

[22] L. Zhang, F. Tian, C. Kadri, B. Xiao, H. Li, L. Pan, H. Zhou, On-line sensor calibration transfer among electronic nose instruments for monitoring volatile organic chemicals in indoor air quality, Sensors and Actuators B: Chemical 160 (1) (2011) 899-909. 
[23] S. Deshmukh, K. Kamde, A. Jana, S. Korde, R. Bandyopadhyay, R. Sankar, N. Bhattacharyya, R. Pandey, Calibration transfer between electronic nose systems for rapid in situ measurement of pulp and paper industry emissions, Analytica chimica acta 841 (2014) 58-67.

[24] K. Yan, D. Zhang, Improving the transfer ability of prediction models for electronic noses, Sensors and Actuators B: Chemical 220 (2015) 115-124.

[25] G. F. Fine, L. M. Cavanagh, A. Afonja, R. Binions, Metal oxide semi-conductor gas sensors in environmental monitoring, Sensors 10 (6) (2010) 5469-5502.

[26] F. Pedregosa, G. Varoquaux, A. Gramfort, V. Michel, B. Thirion, O. Grisel, M. Blondel, P. Prettenhofer, R. Weiss, V. Dubourg, et al., Scikit-learn: Machine learning in python, The Journal of Machine Learning Research 12 (2011) 2825-2830.

[27] M. Pardo, G. Sberveglieri, Classification of electronic nose data with support vector machines, Sensors and Actuators B: Chemical 107 (2) (2005) 730-737.

[28] J. Fonollosa, I. Rodríguez-Luján, M. Trincavelli, A. Vergara, R. Huerta, Chemical discrimination in turbulent gas mixtures with MOX sensors validated by gas chromatography-mass spectrometry, Sensors 14 (10) (2014) 19336-19353.

[29] M. F. Rutolo, D. Iliescu, J. P. Clarkson, J. A. Covington, Early identification of potato storage disease using an array of metal-oxide based gas sensors, Postharvest Biology and Technology 116 (2016) 50-58.

[30] R. N. Feudale, N. A. Woody, H. Tan, A. J. Myles, S. D. Brown, J. Ferré, Transfer of multivariate calibration models: a review, Chemometrics and Intelligent Laboratory Systems 64 (2) (2002) 181-192.

[31] R. Tauler, B. Walczak, S. D. Brown, Comprehensive chemometrics: chemical and biochemical data analysis, Elsevier, 2009.

[32] P. Clifford, D. Tuma, Characteristics of semiconductor gas sensors i. steady state gas response, Sensors and Actuators 3 (0) (1983) 233 - 254. 
[33] P. Clifford, D. Tuma, Characteristics of semiconductor gas sensors ii. transient response to temperature change, Sensors and Actuators 3 (1983) 255-281.

[34] A. Chaiyboun, R. Traute, O. Kiesewetter, S. Ahlers, G. Müller, T. Doll, Modular analytical multicomponent analysis in gas sensor aarrays, Sensors 6 (4) (2006) 270-283.

[35] J. Fonollosa, L. Fernández, R. Huerta, A. Gutiérrez-Gálvez, S. Marco, Temperature optimization of metal oxide sensor arrays using mutual information, Sensors and Actuators B: Chemical 187 (2013) 331-339.

[36] Pls toolbox 7.9. eigenvector research, manson, wa.

[37] Figaro USA, Inc., http://www.figarosensor.com/.

[38] M. Pardo, G. Faglia, G. Sberveglieri, M. Corte, F. Masulli, M. Riani, A time delay neural network for estimation of gas concentrations in a mixture, Sensors and Actuators B: Chemical 65 (1) (2000) 267-269.

[39] S. De Vito, A. Castaldo, F. Loffredo, E. Massera, T. Polichetti, I. Nasti, P. Vacca, L. Quercia, G. Di Francia, Gas concentration estimation in ternary mixtures with room temperature operating sensor array using tapped delay architectures, Sensors and Actuators B: Chemical 124 (2) (2007) 309-316.

[40] M. Ghasemi-Varnamkhasti, M. Aghbashlo, Electronic nose and electronic mucosa as innovative instruments for real-time monitoring of food dryers, Trends in Food Science \& Technology 38 (2) (2014) 158-166.

[41] N. Masson, R. Piedrahita, M. Hannigan, Approach for quantification of metal oxide type semiconductor gas sensors used for ambient air quality monitoring, Sensors and Actuators B: Chemical 208 (2015) 339-345.

[42] J. Fonollosa, S. Sheik, R. Huerta, S. Marco, Reservoir computing compensates slow response of chemosensor arrays exposed to fast varying gas concentrations in continuous monitoring, Sensors and Actuators B: Chemical 215 (2015) 618-629. 
[43] L. Fernandez, S. Guney, A. Gutiérrez-Gálvez, S. Marco, Calibration transfer in temperature modulated gas sensor arrays, Sensors and Actuators B: Chemical (2016) in press.

[44] I. Rodriguez-Lujan, J. Fonollosa, A. Vergara, M. Homer, R. Huerta, On the calibration of sensor arrays for pattern recognition using the minimal number of experiments, Chemometrics and Intelligent Laboratory Systems 130 (2014) 123-134.

[45] E. Martinelli, A. Catini, C. Di Natale, An active temperature modulation of gas sensor based on a self-adaptive strategy, in: Solid-State Sensors, Actuators and Microsystems (TRANSDUCERS \& EUROSENSORS XXVII), 2013 Transducers \& Eurosensors XXVII: The 17th International Conference on, IEEE, 2013, pp. 2045-2048.

[46] R. Gosangi, R. Gutierrez-Osuna, Active temperature modulation of metal-oxide sensors for quantitative analysis of gas mixtures, Sensors and Actuators B: Chemical 185 (2013) 201-210. 


\section{Author Biographies}

J. Fonollosa received his Ph.D. in Electronic Engineering from the University of Barcelona in 2009. His research efforts are focused on the development of algorithmic solutions for chemical detection systems. He has applied chemical sensing to a variety of applications, such as food quality control, fire detection, non-invasive human activity monitoring, and air quality control. He also applied Information Theory to chemical sensing systems. Other strong interests include biologically inspired algorithms, signal recovery systems, and infrared sensing technologies. More at https://jordifonollosa.wordpress.com/

L. Fernandez received a B.S. in Physics (2005), a B.S. in Electronic Engineering (2011), and Ph.D. in Electronic Engineering (2016) from the University of Barcelona. His current research topic is bio-inspired large sensor arrays based on metal oxide gas sensors.

A. Gutierrez-Galvez received the B.E. degree in physics and electrical engineering from the University of Barcelona, Catalonia, Spain, in 1995 and 2000, respectively. He received the Ph.D. degree in computer science from Texas $A \& M$ University, College Station, in 2005. He was a JSPS Post-Doctoral Fellow with the Tokyo Institute of Technology, Tokyo, Japan, in 2006, and came back to the University of Barcelona with a Marie Curie Fellowship. Currently, he is an Assistant Professor with the Department of Electronics, University of Barcelona. His current research interests include biologically inspired processing for gas sensor arrays, computational models of the olfactory systems, pattern recognition, and dynamical systems.

R. Huerta received his Ph.D. from Universidad Autonoma de Madrid in 1994. He is a Research Scientist at the BioCircuits Institute, UC San Diego. Prior his current appointment, he was Associate Professor at the Universidad Autonoma de Madrid (Spain). His areas of expertise include dynamic systems, artificial intelligence, and neuroscience. His work deals with the development algorithms for the discrimination and quantification of complex multidimensional time series, model building to understand the information processing in the brain, and chemical sensing and machine olfaction applications based on bio-inspired technology. Dr. Huerta's research work gathers in a publication record of over 100 articles in peer-reviewed journals at the intersection of computer science, physics, and biology.

S. Marco received his Ph.D. from the University of Barcelona in 1993. He is an Associate Professor at the University of Barcelona and head of the Signal and Information Processing 
for Sensor Systems Lab at the Institute for Bioengineering of Catalonia, Barcelona, Spain. His research concerns the development of signal/data processing algorithmic solutions for smart chemical sensing based in sensor arrays or microspectrometers integrated typically using microsystem technologies. Dr. Marco research has produced over 100 articles in peerreviewed archival journals. 